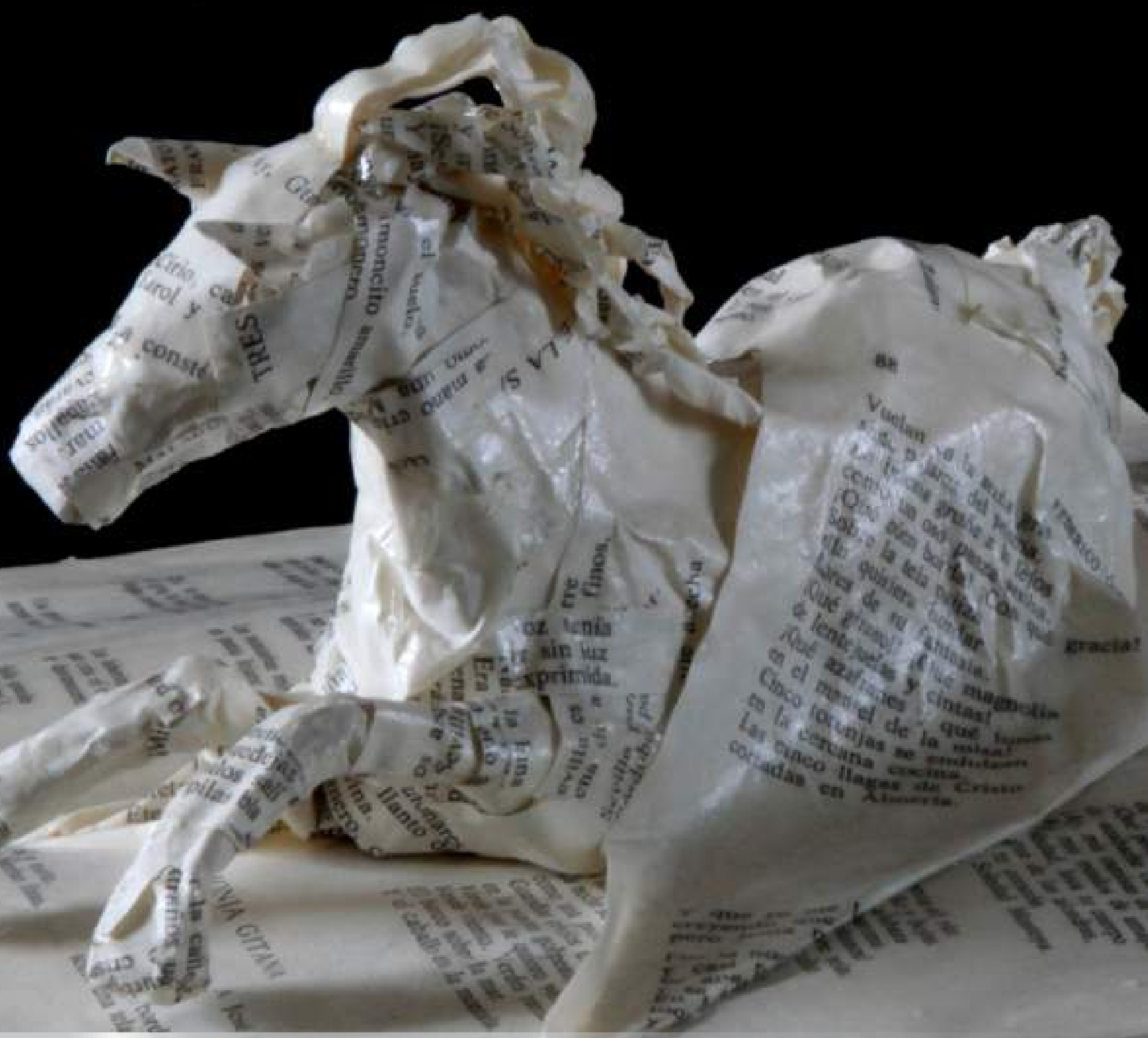

Titulo: "Arco iris negro sobre la noche azul"

Referencia: García Lorca Federico.

Libro de poemas. Ed. Porrúa

Año: 2011

Serie: Libros de Poemas

Técnica: Arte objeto; intervención de libros

Medidas: $28 \times 22 \times 12.5 \mathrm{cms}$.

Foto: Pablo Cuéllar

ISSN 2007-8250 / p.38 - p.47 


\title{
La solución de conflictos y la tutoría académica como predictores del ambiente escolar en alumnos de preparatoria
}

\section{The conflict solution and the academic tutoring as predictors of the school environment in preparatory students}

\author{
Víctor Hugo Almaguer Beltrán \\ Jorge Alfonso Llanas Mendoza
}

\section{Resumen}

Como una manera de contribuir a la formación de los estudiantes, generar una cultura de la paz en nuestra sociedad y tratar de propiciar un clima agradable para los estudiantes, se pretende conocer en qué medida el estudiante de preparatoria percibe la solución de conflictos y la tutoría académica como predictores del ambiente escolar. Para ello, se planteó una investigación aplicada con enfoque explicativo, de corte cuantitativo y transversal. Se administraron 218 encuestas a estudiantes de primer y tercer semestre de la Preparatoria 13 de la Universidad Autónoma de Nuevo León. El modelo indica que dos de los factores son predictores significativos logrando explicar el $12.6 \%$ de la varianza en el clima escolar. Los factores predictores resultaron ser la solución de conflictos por mediación personal $(B=.280, p=.000)$ y la tutoría dedicada y confiable $(B=.167, p=.013)$. En conclusión, ambos factores explican significativamente y con un bajo tamaño del efecto el ambiente escolar. Se recomienda realizar acciones que ayuden a mejorar el clima escolar a través del uso de la mediación como método de la solución de conflictos y la tutoría dedicada y confiable. Socializar los resultados de esta investigación con compañeros puede ayudar a mejorar el clima escolar dentro de la institución educativa.

Palabras clave: métodos, solución, conflicto, mediación, tutoría, escolar.

\begin{abstract}
As a way to contribute to the formation of our students to generate a culture for peace in our society and to promote a pleasant social environment to our students. The objective of this document is to know in what extent high school students perceive conflict resolutions and academic tutoring as predictors of the academic context. This research was applied on the bases of an explicative, quantitative and transversal approach. For this project, 218 surveys were applied to high school students attending first and third semesters of the High School 13, UANL. The survey is composed by demographic data, and three Likert scales; each is focused on specific topics/aspects: academic environment, conflict resolutions and tutoring. This model shows that two factors are significant predictors for explaining the $12.6 \%$ of variance in academic environment. The predictor factors were conflict resolution by personal mediation $(B=.280, p=.000)$ and dedicated and reliable tutoring $(B=.167, p=.013)$. In conclusion, both factors are explained significantly with a low academic environment size effect. It is recommended to take actions to improve the academic environment by using mediation in the conflict solutions and dedicated and reliable tutoring. Socializing the results of this project with colleagues will promote increasing the academic environment at school.
\end{abstract}

Keywords: method, conflict, resolution, mediation, tutoring, scholar. 
$\mathrm{E}$ hablar de la justicia de la paz, es tratar de buscar la mejor manera de solucionar conflictos y esto es algo que se remonta desde hace cientos de años. Según Sanjurjo de Driollet (2010), en Argentina desde 1820 se establecían diversos códigos civiles que se debían cumplir para vivir en sociedad y se designaban a algunas personas llamadas jueces subdelegados, quienes eran los responsables de aplicar la ley, y la "obligación de estos funcionarios menores era de buscar entre las partes concordia, conciliación, paz y arreglo, y sólo una vez agotados estos recursos debían dictar resolución según su conciencia" (Sanjurjo de Driollet, 2010, pp. 399-400).

Ahora bien, la educación tal y como la percibe (Aguilera Portales, 2009) no es sólo la transmisión de conocimientos sino también un cambio de actitudes, hábitos y valores. Una de las preocupaciones actuales que tenemos los maestros es la de tratar de generar una cultura de la paz y la educación para la paz persigue una conciencia ética universal y solidaria, suscitando la participación de todos los miembros de la comunidad social en los problemas colectivos.

El planteamiento del problema es el siguiente: ¿En qué medida la tutoría y la solución de conflictos son predictores significativos del ambiente escolar en los estudiantes de la Preparatoria No 13 de la UANL?

El objetivo general de esta investigación es identificar el efecto de la tutoría y la solución de conflictos en el ambiente escolar y con los objetivos específicos:
- Conocer la percepción del ambiente escolar en la institución. Identificar las características de la tutoría que resultan provechosas a los estudiantes.

-Analizar las estrategias de solución de conflictos utilizadas por los estudiantes.

- Observar las relaciones entre las variables demográficas y los constructos en estudio.

Hipótesis: Los métodos de resolución de conflictos y la tutoría son predictores significativos del ambiente escolar.

Uno de los problemas que se observan en la actualidad en nuestra sociedad es el relacionado con la solución de diversos conflictos, éstos muchas veces son resueltos de un modo que terminan en violencia, y un sector social que se ve influenciado de una manera directa son los jóvenes.

Actualmente se sabe que en nuestro país las diferencias 0 problemas que se presentan, por lo general se arreglan en los juzgados, sin embargo, hay conciencia de que están saturados y la gente que asiste a esos lugares, constantemente se queja de que no hay una solución rápida y eficaz a sus problemas.

Es necesario dar a conocer diversas alternativas para la solución de los problemas que se presentan en el entorno, y los métodos alternos de solución de conflictos son una excelente estrategia para arreglar los problemas, algunos de estos son la mediación, la conciliación y el arbitraje.

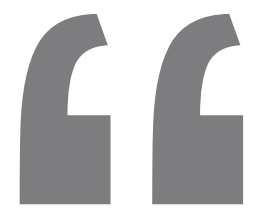

La obligación de estos funcionarios menores era de buscar entre las partes concordia, conciliación, paz y arreglo, y sólo una vez agotados estos recursos debían dictar resolución según su conciencia" (Sanjurjo de Driollet, 2010). 
Los adolescentes, por lo general, tratan de arreglar sus inconvenientes a golpes. Y esto es una preocupación para las escuelas, ya que actualmente se han percibido las consecuencias que tienen los problemas entre los adolescentes en los contextos escolares y fuera de ellos.

Existe un desconocimiento por parte de muchos de los maestros respecto a los métodos alternos de solución de conflictos y los beneficios de los mismos.

El propósito de la presente investigación es conocer el efecto del uso de los métodos para resolver conflictos en la percepción del ambiente escolar en los alumnos de preparatoria y quienes muestran una conducta apropiada no presentan problemas fuertes de violencia dentro de la institución.

Cuando se presenten discusiones de forma cotidiana en los espacios escolares, la mayoría de los problemas se resuelven de manera particular 0 bien son canalizados al departamento de orientación, tutorías 0 a la dirección.

Los maestros como tutores no sólo académicos de sus alumnos, sino también formadores integrales, tienen una injerencia directa con los adolescentes, etapa en la que ellos están en el proceso de formación y sus acciones y consejos llegan a ser de gran influencia para ellos, de ahí la importancia de conocer estos métodos.

La mediación según Steele Garza (2009), funciona como instrumento para la gestión positiva de los con- flictos, siendo éstos los efectos en las personas y en la comunidad a sus eventuales acuerdos que refuerzan la cohesión social, protegen las relaciones e incrementan el nivel de pertenencia y compromiso de las personas con la obtención y mantenimiento del bien común.

Además la mediación es como una actuación en el interior de un sistema de relaciones y de interacciones entre los actores sociales, cada uno de los cuales es portador de competencias y saberes diversificados.

Es, antes que una técnica dirigida a afrontar específicas situaciones conflictivas, una condición constitutiva de las relaciones sociales, como lo es por otro lado el conflicto.

Según Gorjón Gómez (2008), la mediación es la aplicación de técnicas específicas para lograr que las partes lleguen a comunicarse e identifiquen específicamente su problema, de modo que puedan encontrar alternativas para resolverlo atendiendo siempre las características del conflicto.

Uno de los lugares de gran influencia para la formación del adolescente es la escuela, ya que ésta constituye el "marco más adecuado para ampliar y enriquecer las relaciones interpersonales ofrecidas por otros contextos (familia, sociedad, etcétera) y para alcanzar, a través del grupo de iguales, el desarrollo cognitivo, afectivo y social de cada uno de sus miembros" (González-Tejero et al., 2007, p. 125).

\section{El Estudio Estatal de Convivencia} Escolar encuentra que "las conductas contrarias a la convivencia están estrechamente relacionadas con el

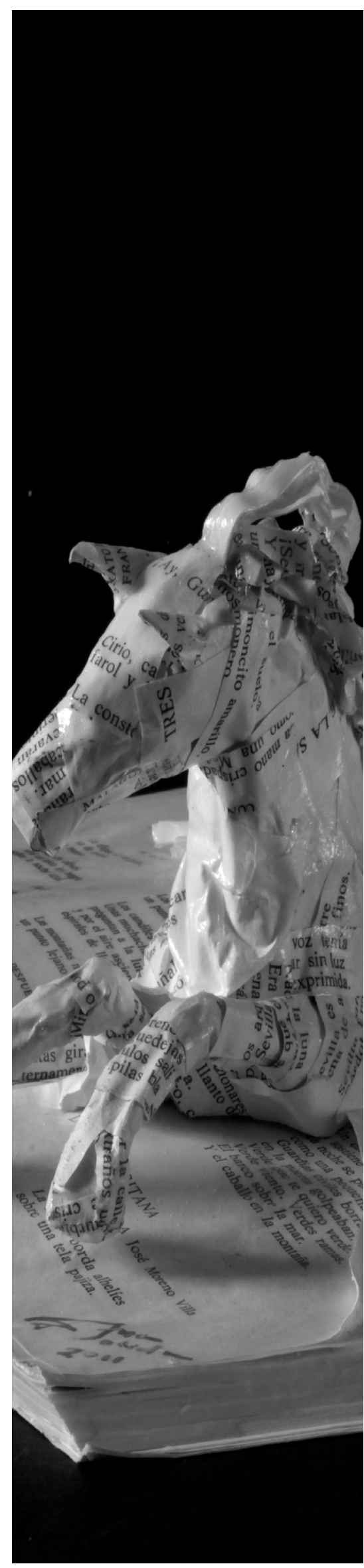

Presencia Universitaria 


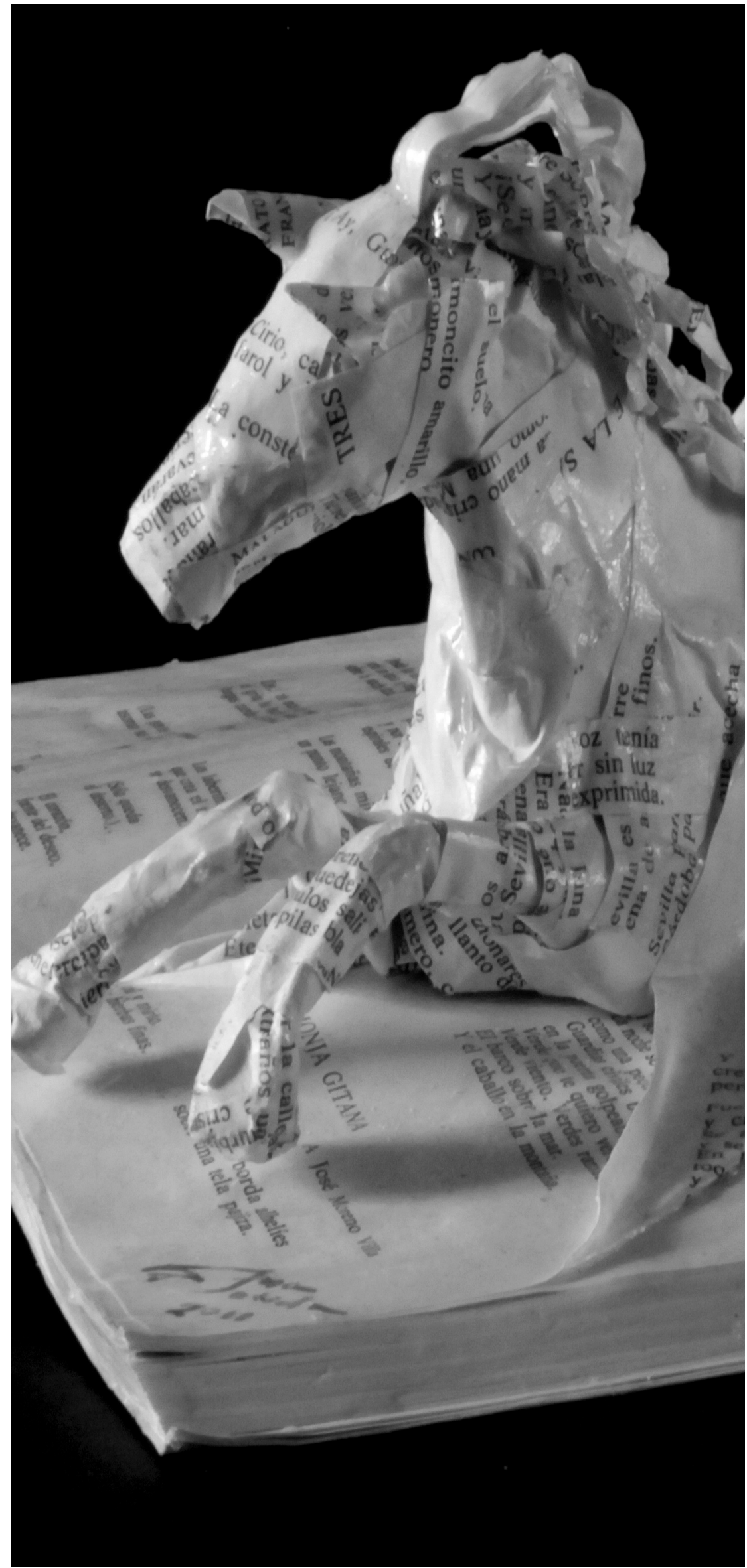

consumo de drogas, dicha conducta parece formar parte de los trastornos de externalización" (Díaz-Aguado Jalón, y Martín Seoane, 2011, p. 255).

El docente desarrolla una función fundamental en los procesos de aprendizaje de los estudiantes, tanto sus prácticas pedagógicas como su dimensión personal; resultan ser facilitadores u obstaculizadores del desempeño de los estudiantes (Valencia et al., 2014).

Se debe pensar la mejor manera en la que podemos influenciar en los estudiantes, en cuanto a su formación y desempeño, ya que el papel que desempeñan con ellos es fundamental, para esto es necesario contar con un amplio criterio y una buena disposición para las actualizaciones docentes que se presenten, así como una práctica profesional de su labor educativa.

Rodríguez (citado en Reyes Rodríguez e Izquierdo Dorta, 2012) menciona las siguientes cinco dimensiones del trabajo del tutor: académico, profesional, personalidad, político ideológica y la socioeconómica-familiar.

Son muchos los aspectos y responsabilidades que adquieren los tutores y no sólo tiene que ver con lo académico, sino que va más allá de esto, por lo que para el estudiante la función que realizan como maestros tutores puede ser fundamental en su formación personal y profesional.

Los mecanismos alternativos son procedimientos distintos a la justicia ordinaria que permiten prevenir, abordar y solucionar controversias de manera voluntaria y colaborativa. 
Éstos se implementarán en forma presencial 0 , en los casos en que resulte procedente, a distancia mediante el empleo de tecnologías de la información y la comunicación.

La población estudiada esta compuesta de 480 alumnos de la escuela Preparatoria No. 13 de la UANL. Se agrupan en cuatro bachilleratos: progresivo en inglés, en francés, general y técnico.

Para seleccionar a los participantes del estudio se utilizó el método de muestreo accidental por racimos y estratificado según el tipo de bachillerato. Se escogieron los grupos con base en la disponibilidad de horarios y aprobación de los maestros. La muestra quedó constituida por 218 sujetos que corresponde al $45 \%$ de la población.

Se administró el instrumento de investigación a 218 estudiantes de preparatoria. La distribución del género es muy equilibrada ya que el $52 \%$ son mujeres y la edad de los estudiantes es mayormente de 15 y 16 años $(92 \%)$ de los cuales la mayoría vive con ambos padres (86\%).

Más de la mitad estudian en el bachillerato general (56\%) y consideran que su conducta en el nivel secundario fue buena (38\%) o muy buena ( $47 \%)$.

El instrumento de investigación se compone de tres tipos de variables: -Variables demográficas: Edad, género, con quién vive, nivel académico de sus padres, nivel socioeconómico, su conducta en el nivel educativo anterior, medio de transporte para ir a la escuela y bachillerato que estudia.
-Variables predictoras: La tutoría y la solución de conflictos.

-Variable criterio: Ambiente escolar.

La escala del ambiente escolar incluye 14 declaraciones para ser valoradas por los estudiantes. La escala de solución de conflictos se compone de 34 ítems y la de tutoría de 17.

En todos los casos la escala utilizada incluye cinco puntos que van desde el desacuerdo (1) hasta el acuerdo (5). En relación a la tutoría también se les preguntó por la persona a la cual acuden de manera preferente para recibir apoyo u orientación, siendo las opciones: mi tutor, otro maestro 0 algún directivo. El procedimiento seguido para la realización de este proyecto de investigación, se compone de las siguientes etapas:

1. Identificación de una problemática: La solución de conflictos en ambientes escolares y la formación de los estudiantes en este aspecto.

2. Identificación del problema: Selección de constructos relacionados con la solución de conflictos.

Se escogió la tutoría como un elemento asociado de tal forma que ayude a explicar el ambiente escolar.

En estas dos primeras etapas se analizó desde la perspectiva teórica y se tomaron decisiones en la selección de los constructos con base en la experiencia y asesoría.

3. Establecimiento de la metodología. Se tomaron decisiones para tener un acercamiento a la realidad y obtener información empírica.

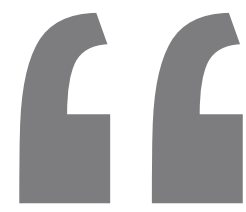

En estas dos

primeras etapas se analizó desde la perspectiva teórica y se tomaron decisiones en

la selección de los constructos con base en la experiencia y asesoría". 


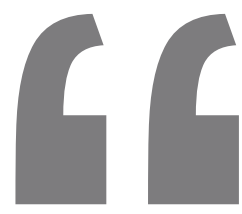

\section{Las tres subescalas} identificadas son:

\section{Tutoría dedicada}

y confiable con seis

ítems, la tutoría

orientadoray

comprensiva

con cinco ítems

y la tutoría

\section{castigadora}

y negativa

también con cinco

ítems".
4. Aplicación y análisis de los datos recolectados. Uso de herramientas estadísticas para obtener evidencias de la respuesta a la pregunta de investigación.

5. Presentación del informe. Revisión general del documento y establecimiento de conclusiones y recomendaciones.

Se identificaron dos factores que explican el $42.4 \%$ de la varianza total. Los factores de la escala se orientan hacia el ambiente escolar derivado de las relaciones con los profesores y el otro derivado del clima social en la escuela.

El factor de los profesores consta de 6 items en el instrumento original y sólo uno de ellos no se agrupó conforme a la teoría.

El ítem 8, el cual valora la felicitación del estudiante por parte del profesor cuando hace un buen trabajo, muestra una carga muy baja en el factor correspondiente $(r=.174)$ y es percibido más como un elemento del clima social de la escuela $(r=.451)$.

El segundo factor se compone originalmente de ocho ítems, pero tres de ellos muestran cargas muy bajas; el ítem 1 sobre encontrar ayuda en la escuela cuando tiene una emergencia ( $r=-057)$, el ítem 12 que valora la escuela como un lugar seguro $(r=$ .284) y el considerar la escuela como un lugar ordenado y limpio (ítem 5 , $r=.213$ ). La escala de solución de conflictos fue elaborada por el investigador.

Consta de seis factores: mediación (Med), conciliación (Coc), confronta- miento (Cof), evitación (Evi), colaboración (Col) y negación (Neg).

La escala original constaba de 34 ítems. Al hacer los análisis de validación, la escala se redujo a 29. En este caso la adecuación muestral también es satisfactoria (KMO $=.816)$ y la matriz de correlaciones difiere de la matriz identidad según la esfericidad de Bartlett (Chi-cuadrado (561) $=2807.940, \mathrm{p}=.000)$. Respecto a la mediación se percibió una subdivisión de los ítems.

Cuatro ítems que valoran la postura 0 actitud de los sujetos involucrados en el conflicto (escuchar, respetar, aclarar, empatía y apertura) y tres ítems asociados con la función del mediador (imparcialidad, ayudador y orientador) los cuales se agruparon con el factor de conciliación.

La mediación personal se compone de cuatro ítems (alfa $=.764$ ), la mediación mediador consta de tres ítems (alfa $=.743$ ), la evitación tiene seis ítems (alfa $=.749$ ), la conciliación incluye cuatro ítems (alfa $=.826$ ), la colaboración se compone de cuatro ítems (alfa $=.664$ ), la negación con tres ítems (alfa $=.601$ ) y la confrontación con cinco ítems (alfa $=.833$ ).

La escala de tutoría fue creada por el investigador. En el análisis factorial (KMO $=.830$, Chi-Cuadrada (136) $=1304.710, p=.000$ ) se identificaron tres subescalas que explican el $50.2 \%$ de la varianza total. Las tres subescalas identificadas son: Tutoría dedicada y confiable con seis ítems, la tutoría orientadora y comprensiva con cinco ítems y la tutoría castigadora y negativa también con cinco ítems. 
La confiabilidad de la tutoría dedicada y confiable es de .889, para la tutoría orientadora y comprensiva es de .647 y para la tutoría castigadora y negativa resultó en .589 .

El proceso de investigación realizado con respecto al ambiente escolar, la solución de conflictos y la tutoría en estudiantes de bachillerato de la Preparatoria No 13 de la UANL, durante el curso escolar 2016-2017, permite establecer las siguientes conclusiones:

1. Los factores predictores del ambiente escolar son la solución de conflictos por mediación personal y la tutoría dedicada y confiable. Explican el $13 \%$ de la varianza, según la prueba de hipótesis.

2. El poder predictivo de las variables es mayor en las mujeres, un $27 \%$ mientras que en los hombres es del $6 \%$.

3. Los instrumentos creados para valorar la solución de conflictos y la tutoría, muestran niveles de calidad aceptable. El de solución de conflictos valora los procesos de mediación, conciliación, confrontación, evitación, colaboración y negación. El de tutoría valora tres tipos; tutoría dedicadaconfiable, orientadora-comprensiva y castigadora-negativa.

4. Las estrategias que más se utilizan para resolver conflictos son la mediación y la colaboración. Utilizan de manera regular las estrategias de evitación, conciliación y negación. Y casi nunca utilizan la confrontación.

5. En cuanto a tutorías, los estudiantes prefieren más la que es dedicada y confiable seguida de la tutoría comprensiva y orientadora.

6. Las mujeres tienen una percepción del ambiente escolar peor que los hombres.

7. Las personas que viven con ambos padres perciben la mediación personal de una manera más positiva y esto les ayuda a desarrollar más las características de dicha mediación.

8. El nivel académico de los padres de familia es un factor de influencia para usar la colaboración en la solución de conflictos. 


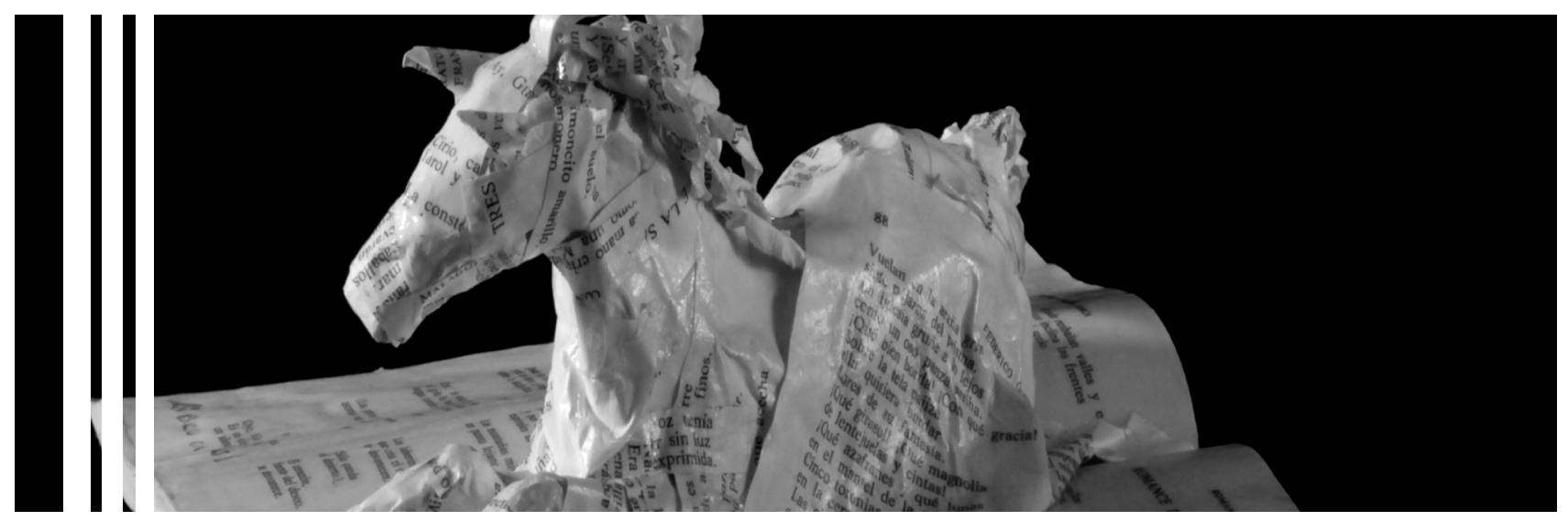

\section{Referencias}

Díaz-Aguado Jalón, M. J. y Martín Seoane, G. (2011). Convivencia y aprendizaje escolar en la adolescencia desde una perspectiva de género. Psicothema, 23(2), España.

Gorjón Gómez, F. J. (2008). Métodos alternativos de solución de conflictos. México: Oxford University Press.

Sanjurjo de Driollet,I. (2010).La institución de la justicia de paz: la resolución de controversias vecinales de menor cuantía en materia civil y comercial (San Rafael, Mendoza, 1900-1916). Revista de estudios histórico-jurídicos, 32, Chile.

Reyes Rodríguez, R. e Izquierdo Dorta, C. (2012). El plan de acción integral tutorial. Su papel en la nueva universidad cubana. Pedagogía Universitaria, 17(2), Cuba.

Steele Garza,J.G. (2009). Mediación y conciliación en la Procuraduría General de Justicia en el Estado de Nuevo León. En Gorjón Gómez, F.J.(Editor), Mediación y arbitraje. Leyes comentadas y concordadas del Estado de Nuevo León, México: Editorial Porrúa.

Valencia, L. I., Galeano, A. y Joven, K. (2014). Estilos de enseñanza de los docentes: una apuesta por el desempeño académico de los estudiantes en la Educación Superior. Revista Mexicana de Orientación Educativa, 11(26), México.

Compilación Legislativa del Estado de Nuevo León (2017). Ley de Mecanismos Alternos para la Solución de Controversias del Estado de Nuevo León. 


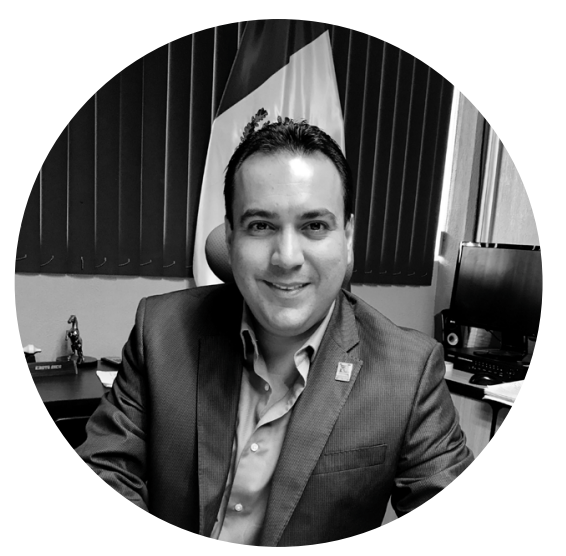

\section{Víctor Hugo Almaguer Beltrán}

Es Licenciado en Educación Secundaria en la Especialidad de Lengua Extranjera (Inglés), egresado de la Escuela Normal Superior "Profr. Moisés Sáenz Garza". Tiene una maestría en Educación Media en la Especialidad de Idiomas de la Escuela de Graduados de la Normal Superior "Profr. Moisés Sáenz Garza" y una maestría en Métodos Alternos de Solución de Controversias de la Facultad de Derecho y Criminología de la UANL. Tiene un Doctorado en Ciencias de la Educación por la Universidad Autónoma de Coahuila. Además de diferentes cursos y diplomados en la Enseñanza del Inglés. Actualmente es Subdirector Académico de la Preparatoria 13 de la UANL y catedrático de la Escuela Normal Superior "Profr. Moisés Sáenz Garza" y de la Escuela Normal Básica "Profr. Serafín Peña".

Correo electrónico:

victorhalmaguer@hotmail.com

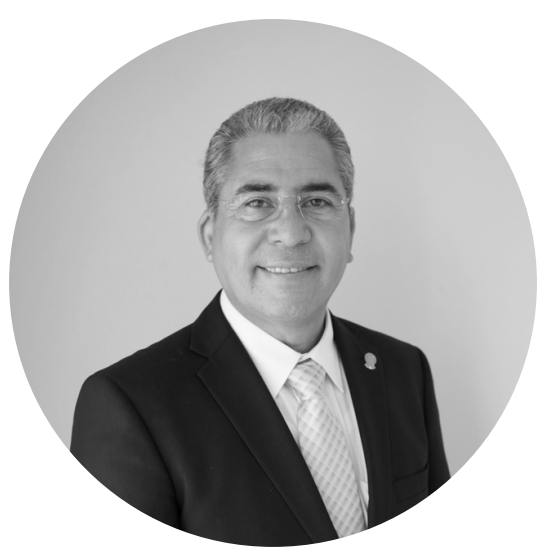

\section{Jorge Alfonso Llanas Mendoza}

Es Licenciado en Arquitectura, egresado de la Facultad de Arquitectura de la UANL. Tiene una maestría en Enseñanza de las Ciencias con la especialidad en Matemáticas de la Facultad de Filosofía y Letras y la Facultad de Matemáticas de la UANL. Cuenta con un Doctorado en Ciencias de la Educación por la Universidad Autónoma de Coahuila. Además de contar con diversos cursos y diplomados en el área de Matemáticas, Física, Calidad, Administración, entre otros. Tienen 32 años de Servicio en la Educación dentro de la UANL y actualmente es el Director de la Preparatoria 13 de la UANL.

Correo electrónico:

elarquillanas@hotmail.com 